\title{
LANDFILL-GAS-TO-ENERGY PROJECTS: AN ANALYSIS OF NET PRIVATE AND SOCIAL BENEFITS
}

\author{
Supplemental Materials
}

Table A1: Model Results for West Lake Landfill

\begin{tabular}{|l|c|c|c|}
\hline \multicolumn{1}{|c|}{ WEST LAKE } & IC Engine & Gas Turbine & Steam Turbine \\
\hline Average Landfill Gas Generation (mmcf/yr) & 1,888 & 1,888 & 1,888 \\
\hline Average Generation Potential (MWh/yr) & 69,925 & 57,440 & 52,225 \\
\hline Average Generation (MWh/yr) & 60,665 & 56,495 & 51,235 \\
\hline Number of Units & 3 & 1 & 3 \\
\hline Unit Capacity (MW) & 3 & 10 & 3 \\
\hline Capital Costs (thousand \$) & $\$ 8,270$ & $\$ 9,690$ & $\$ 9,135$ \\
\hline O\&M Costs (\$/kWh) & $\$ 0.009$ & $\$ 0.006$ & $\$ 0.005$ \\
\hline Private Net Present Value (thousand 1999\$) & $\$ 5,695$ & $\$ 6,300$ & $\$ 5,360$ \\
\hline
\end{tabular}

Table A2: Model Results for West County Landfill

\begin{tabular}{|l|c|c|c|}
\hline \multicolumn{1}{|c|}{ WEST COUNTY } & IC Engine & Gas Turbine & Steam Turbine \\
\hline Average Landfill Gas Generation (mmef/yr) & 1,075 & 1,075 & 1,075 \\
\hline Average Generation Potential (MWh/yr) & 39,820 & 32,710 & 29,740 \\
\hline Average Generation (MWh/yr) & 38,580 & 31,875 & 29,740 \\
\hline Number of Units & 2 & 1 & 2 \\
\hline Unit Capacity (MW) & 3 & 5 & 3 \\
\hline Capital Costs (thousand \$) & $\$ 5,515$ & $\$ 5,050$ & $\$ 6,090$ \\
\hline O\&M Costs (\$/kWh) & $\$ 0.009$ & $\$ 0.006$ & $\$ 0.007$ \\
\hline Private Net Present Value (thousand 1999\$) & $\$ 3,190$ & $\$ 2,735$ & $\$ 1,250$ \\
\hline
\end{tabular}

Table A3: Model Results for Modern Landfill

\begin{tabular}{|l|c|c|c|}
\hline \multicolumn{1}{|c|}{ MODERN } & IC Engine & Gas Turbine & Steam Turbine \\
\hline Average Landfill Gas Generation (mmcf/yr) & 744 & 744 & 744 \\
\hline Average Generation Potential (MWh/yr) & 27,555 & 22,630 & 20,580 \\
\hline Average Generation (MWh/yr) & 25,195 & 22,630 & 18,850 \\
\hline Number of Units & 5 & 1 & 1 \\
\hline Unit Capacity (MW) & 0.8 & 5 & 3 \\
\hline Capital Costs (thousand \$) & $\$ 4,010$ & $\$ 5,050$ & $\$ 3,045$ \\
\hline O\&M Costs (\$/kWh) & $\$ 0.01$ & $\$ 0.006$ & $\$ 0.008$ \\
\hline Private Net Present Value (thousand 1999\$) & $\$ 1,210$ & $\$ 815$ & $\$ 840$ \\
\hline
\end{tabular}

Table A4: Emissions and Valuations from West Lake Landfill 


\begin{tabular}{|l|c|c|c|c|c|}
\hline \multicolumn{1}{|c|}{ WEST LAKE } & Uncontrolled & Flare & IC Engine & Gas Turbine & $\begin{array}{c}\text { Steam } \\
\text { Turbine }\end{array}$ \\
\hline Average Annual Methane Emissions (tons) & 19,284 & 2,893 & 2,893 & 2,893 & 2,893 \\
\hline Average Annual CO $\mathbf{O}_{2}$ Emissions (tons) & 53,031 & 98,108 & 98,108 & 98,108 & 98,108 \\
\hline Average Annual SO $\mathbf{S}_{2}$ Emissions (tons) & 0 & 0.12 & 0.12 & 0.12 & 0.12 \\
\hline Average Annual NO $\mathbf{x}$ Emissions (tons) & 0 & 16.05 & 100.31 & 34.91 & 13.24 \\
\hline Average Annual CO Emissions (tons) & 0 & 300.94 & 188.59 & 92.29 & 2.29 \\
\hline Average Annual PM Emissions (tons) & 0 & 6.82 & 19.26 & 8.83 & 3.29 \\
\hline $\begin{array}{l}\text { Net Present Value of Emission Costs } \\
\text { (thousand 1999\$) }\end{array}$ & $\$ 80,500$ & $\$ 31,045$ & $\$ 34,170$ & $\$ 30,410$ & $\$ 28,635$ \\
\hline $\begin{array}{l}\text { Emission Savings Compared to Uncontrolled } \\
\text { Emissions (thousand 1999\$) }\end{array}$ & $\$ 0$ & $\$ 49,460$ & $\$ 46,335$ & $\$ 50,095$ & $\$ 51,870$ \\
\hline $\begin{array}{l}\text { Emission Savings Compared to Flare } \\
\text { Emissions (thousand 1999\$) }\end{array}$ & $-\$ 49,460$ & $\$ 0$ & $-\$ 3,120$ & $\$ 635$ & $\$ 2,410$ \\
\hline
\end{tabular}

Table A5: Emissions and Valuations from West County Landfill

\begin{tabular}{|l|c|c|c|c|c|}
\hline \multicolumn{1}{|c|}{ WEST COUNTY } & Uncontrolled & Flare & IC Engine & Gas Turbine & $\begin{array}{c}\text { Steam } \\
\text { Turbine }\end{array}$ \\
\hline Average Annual Methane Emissions (tons) & 10,982 & 1,647 & 1,647 & 1,647 & 1,647 \\
\hline Average Annual CO $\mathbf{O}_{2}$ Emissions (tons) & 30,199 & 55,869 & 55,869 & 55,869 & 55,869 \\
\hline Average Annual $\mathbf{S O}_{\mathbf{2}}$ Emissions (tons) & 0 & 0.07 & 0.07 & 0.07 & 0.07 \\
\hline Average Annual NO $\mathbf{X}_{\mathbf{x}}$ Emissions (tons) & 0 & 9.14 & 57.12 & 19.88 & 7.54 \\
\hline Average Annual CO Emissions (tons) & 0 & 171.37 & 107.39 & 52.55 & 1.30 \\
\hline Average Annual PM Emissions (tons) & 0 & 3.88 & 10.97 & 5.03 & 1.87 \\
\hline $\begin{array}{l}\text { Net Present Value of Emission Costs } \\
\text { (thousand 1999\$) }\end{array}$ & $\$ 42,910$ & $\$ 16,550$ & $\$ 18,215$ & $\$ 16,210$ & $\$ 15,260$ \\
\hline $\begin{array}{l}\text { Emission Savings Compared to } \\
\text { Uncontrolled Emissions (thousand 1999\$) }\end{array}$ & $\$ 0$ & $\$ 26,365$ & $\$ 24,670$ & $\$ 26,700$ & $\$ 27,650$ \\
\hline $\begin{array}{l}\text { Emission Savings Compared to Flare } \\
\text { Emissions (thousand 1999\$) }\end{array}$ & $-\$ 26,365$ & $\$ 0$ & $-\$ 1,665$ & $\$ 400$ & $\$ 1,285$ \\
\hline
\end{tabular}

Table A6: Emissions and Valuations from Modern Landfill

\begin{tabular}{|l|c|c|c|c|c|}
\hline \multicolumn{1}{|c|}{ MODERN } & Uncontrolled & Flare & IC Engine & Gas Turbine & $\begin{array}{c}\text { Steam } \\
\text { Turbine }\end{array}$ \\
\hline Average Annual Methane Emissions (tons) & 7,599 & 1,140 & 1,140 & 1,140 & 1,140 \\
\hline Average Annual CO $\mathbf{O}_{2}$ Emissions (tons) & 20,897 & 38,659 & 38,659 & 38,659 & 38,659 \\
\hline Average Annual SO Emissions (tons) & 0 & 0.05 & 0.05 & 0.05 & 0.05 \\
\hline Average Annual NO $\mathbf{x}$ Emissions (tons) & 0 & 6.32 & 39.53 & 13.76 & 5.22 \\
\hline Average Annual CO Emissions (tons) & 0 & 118.58 & 74.31 & 36.37 & 0.90 \\
\hline Average Annual PM Emissions (tons) & 0 & 2.69 & 7.59 & 3.48 & 1.30 \\
\hline $\begin{array}{l}\text { Net Present Value of Emission Costs } \\
\text { (thousand 1999\$) }\end{array}$ & $\$ 32,080$ & $\$ 12,370$ & $\$ 13,615$ & $\$ 12,120$ & $\$ 11,410$ \\
\hline $\begin{array}{l}\text { Emission Savings Compared to } \\
\text { Uncontrolled Emissions (thousand 1999\$) }\end{array}$ & $\$ 0$ & $\$ 19,710$ & $\$ 18,465$ & $\$ 19,960$ & $\$ 20,670$ \\
\hline $\begin{array}{l}\text { Emission Savings Compared to Flare } \\
\text { Emissions (thousand 1999\$) }\end{array}$ & $-\$ 19,710$ & $\$ 0$ & $-\$ 1,245$ & $\$ 255$ & $\$ 960$ \\
\hline
\end{tabular}

Table A7: Emission Offsets from Electricity Production at West Lake Landfill 


\begin{tabular}{|l|c|c|c|}
\hline \multicolumn{1}{|c|}{ WEST LAKE } & IC Engine & Gas Turbine & Steam Turbine \\
\hline Average Annual $\mathbf{C O}_{2}$ Emission Offsets (tons) & 37,521 & 34,941 & 31,688 \\
\hline Average Annual $\mathbf{S O}_{2}$ Emission Offsets (tons) & 157.73 & 146.88 & 133.21 \\
\hline Average Annual $\mathbf{N O}_{\mathbf{x}}$ Emission Offsets (tons) & 81.90 & 76.27 & 69.17 \\
\hline $\begin{array}{l}\text { Net Present Value of Emission Offsets (thousand } \\
\text { 1999\$) }\end{array}$ & $\$ 11,005$ & $\$ 11,240$ & $\$ 10,170$ \\
\hline
\end{tabular}

Table A8: Emission Offsets from Electricity Production at West County Landfill

\begin{tabular}{|l|c|c|c|}
\hline \multicolumn{1}{|c|}{ WEST COUNTY } & IC Engine & Gas Turbine & Steam Turbine \\
\hline Average Annual $\mathbf{C O}_{2}$ Emission Offsets (tons) & 23,863 & 19,714 & 18,394 \\
\hline Average Annual $\mathbf{S O}_{2}$ Emission Offsets (tons) & 100.31 & 82.87 & 77.32 \\
\hline Average Annual $\mathbf{N O}_{\mathbf{x}}$ Emission Offsets (tons) & 52.09 & 43.03 & 40.15 \\
\hline $\begin{array}{l}\text { Net Present Value of Emission Offsets (thousand } \\
\text { 1999\$) }\end{array}$ & $\$ 7,200$ & $\$ 5,965$ & $\$ 5,620$ \\
\hline
\end{tabular}

Table A9: Emission Offsets from Electricity Production at Modern Landfill

\begin{tabular}{|l|c|c|c|}
\hline \multicolumn{1}{|c|}{ MODERN } & IC Engine & Gas Turbine & Steam Turbine \\
\hline Average Annual $\mathbf{C O}_{2}$ Emission Offsets (tons) & 15,584 & 13,999 & 11,660 \\
\hline Average Annual $\mathbf{S O}_{2}$ Emission Offsets (tons) & 65.51 & 58.85 & 49.02 \\
\hline Average Annual $\mathrm{NO}_{\mathbf{x}}$ Emission Offsets (tons) & 34.02 & 30.55 & 25.45 \\
\hline $\begin{array}{l}\text { Net Present Value of Emission Offsets (thousand } \\
\text { 1999\$) }\end{array}$ & $\$ 4,700$ & $\$ 4,620$ & $\$ 3,520$ \\
\hline
\end{tabular}

The following tables show the results of the analysis for IC engines and gas turbines using the Michigan DEQ emission factors instead of the AP-42 emission factors. Tables A10 to A13 show the potential emissions and emission valuations, and Tables A14 to A15 show the total financial results for the projects.

Table A10: Michigan DEQ Emissions for West Lake Landfill.

\begin{tabular}{|l|c|c|}
\hline \multicolumn{1}{|c|}{ WEST LAKE } & IC Engine & Gas Turbine \\
\hline Average Annual $\mathrm{Methane} \mathrm{Emissions} \mathrm{(tons)}_{\mathrm{C}}$ & 2,893 & 2,893 \\
\hline Average Annual $\mathrm{CO}_{2}$ Emissions (tons) & 98,108 & 98,108 \\
\hline Average Annual $\mathrm{SO}_{2}$ Emissions (tons) & 0.48 & 0.48 \\
\hline Average Annual $\mathrm{NO}_{\mathrm{x}}$ Emissions (tons) & 100.31 & 34.91 \\
\hline Average Annual CO Emissions (tons) & 320.20 & 92.29 \\
\hline Average Annual PM Emissions (tons) & 8.02 & 35.31 \\
\hline Net Present Value of Emission Costs (thousand 1999\$) & $\$ 34,450$ & $\$ 31,960$ \\
\hline $\begin{array}{l}\text { Emission Savings Compared to Uncontrolled Emissions (thousand } \\
1999 \$ \text { ) }\end{array}$ & $\$ 46,050$ & $\$ 48,545$ \\
\hline Emission Savings Compared to Flare Emissions (thousand 19999) & $-\$ 3,405$ & $-\$ 915$ \\
\hline
\end{tabular}


Table A11: Michigan DEQ Emissions for West County Landfill.

\begin{tabular}{|l|c|c|}
\hline \multicolumn{1}{|c|}{ WEST COUNTY } & IC Engine & Gas Turbine \\
\hline Average Annual Methane Emissions (tons) & 1,647 & 1,647 \\
\hline Average Annual $\mathrm{CO}_{2}$ Emissions (tons) & 55,869 & 55,869 \\
\hline Average Annual $\mathrm{SO}_{2}$ Emissions (tons) & 0.27 & 0.27 \\
\hline Average Annual NO $\mathrm{N}_{\mathrm{x}}$ Emissions (tons) & 57.12 & 19.88 \\
\hline Average Annual CO Emissions (tons) & 182.34 & 52.55 \\
\hline Average Annual PM Emissions (tons) & 4.57 & 20.11 \\
\hline Net Present Value of Emission Costs (thousand 1999\$) & $\$ 18,365$ & $\$ 17,035$ \\
\hline Emission Savings Compared to Uncontrolled Emissions (thousand 1999\$) & $\$ 24,550$ & $\$ 25,875$ \\
\hline Emission Savings Compared to Flare Emissions (thousand 1999\$) & $-\$ 1,815$ & $-\$ 485$ \\
\hline
\end{tabular}

Table A12: Michigan DEQ Emissions for Modern Landfill.

\begin{tabular}{|l|c|c|}
\hline \multicolumn{1}{|c|}{ MODERN } & IC Engine & Gas Turbine \\
\hline Average Annual Methane Emissions (tons) & 1,140 & 1,140 \\
\hline Average Annual $\mathrm{CO}_{2}$ Emissions (tons) & 38,659 & 38,659 \\
\hline Average Annual $\mathrm{SO}_{2}$ Emissions (tons) & 0.19 & 0.19 \\
\hline Average Annual NO ${ }_{x}$ Emissions (tons) & 39.53 & 13.76 \\
\hline Average Annual CO Emissions (tons) & 126.17 & 36.37 \\
\hline Average Annual PM Emissions (tons) & 3.16 & 13.91 \\
\hline Net Present Value of Emission Costs (thousand 1999\$) & $\$ 13,730$ & $\$ 12,735$ \\
\hline $\begin{array}{l}\text { Emission Savings Compared to Uncontrolled Emissions (thousand } \\
1999 \$)\end{array}$ & $\$ 18,350$ & $\$ 19,345$ \\
\hline Emission Savings Compared to Flare Emissions (thousand 1999\$) & $-\$ 1,355$ & $-\$ 365$ \\
\hline
\end{tabular}

Table A13: Total NPV for West Lake Landfill Using the Michigan DEQ Emission Factors (all values in thousand 1999\$).

\section{WEST LAKE}

IC Engine

Gas Turbine

\begin{tabular}{lcc} 
Private Net Present Value & $\$ 5,695$ & $\$ 6,300$ \\
Emission Savings Compared to Flare Emissions & $-\$ 3,405$ & $-\$ 915$ \\
\hline Net Present Value Without Emission Offsets & $\$ 2,290$ & $\$ 5,385$ \\
Net Present Value of Emission Offsets & $\$ 11,005$ & $\$ 11,240$ \\
\hline Total Social Net Present Value & $\$ 13,295$ & $\$ 16,625$ \\
&
\end{tabular}

\section{WEST COUNTY}

Private Net Present Value

Emission Savings Compared to Flare Emissions

Net Present Value Without Emission Offsets

Net Present Value of Emission Offsets

Total Social Net Present Value
IC Engine

Gas Turbine

$\$ 3,190 \quad \$ 2,735$

$-\$ 1,815$

$-\$ 485$

$\$ 1,375 \quad \$ 2,250$

$\$ 7,200$

$\$ 5,965$

$\$ 8,575$

$\$ 8,215$ 
Table A15: Total NPV for Modern Landfill Using the Michigan DEQ Emission Factors (all values in thousand 1999\$).

\begin{tabular}{lcc}
\multicolumn{1}{c}{ MODERN } & IC Engine & Gas Turbine \\
\hline Private Net Present Value & $\$ 1,210$ & $\$ 815$ \\
Emission Savings Compared to Flare Emissions & $-\$ 1,355$ & $-\$ 365$ \\
\hline Net Present Value Without Emission Offsets & $-\$ 145$ & $\$ 450$ \\
Net Present Value of Emission Offsets & $\$ 4,700$ & $\$ 4,620$ \\
\hline Total Social Net Present Value & $\$ 4,555$ & $\$ 5,070$
\end{tabular}

